\title{
Coach my Career - ein starkes Zeichen gelebter Solidarität
}

\section{Jürg Unger-Köppel}

Dr. med., Mitglied des FMH-Zentralvorstandes, Departementsverantwortlicher Stationäre Versorgung und Tarife

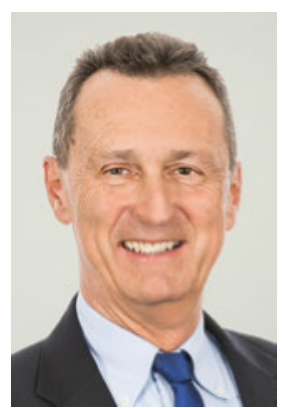

«Ärzte sind keine Teamplayer», «Ärzte können nicht zusammenarbeiten», "Ärzte müssen immer alles bestimmen», «Das ärztliche Weltbild ist stark hierarchisch geprägt» oder «Schon im Studium herrscht ein grosser Konkurrenzkampf» - Wem von uns wurden diese Sätze noch nie um die Ohren geschlagen? Aber halt: Haben wir nicht gerade im Studium immer in Gruppen zusammen gelernt? Treffen sich nicht «Staatsgruppen» oft bis ins hohe Alter? Sprechen wir nicht eine gemeinsame Sprache, die uns prägt und die Verständigung untereinander leichtmacht? Die obgenannten Vorurteile stimmen also so nicht ganz. Trotzdem halten sie sich hartnäckig. Aber von irgendwoher kommen solche Vorurteile.

Neue Kanäle wie YouTube können langjährige Erfahrungen und gesammeltes Wissen eines Menschen nicht ersetzen.

Die meisten von uns wurden nach dem Studium zuerst beruflich in den Spitälern geprägt. Plötzlich ist man in der Klinik mit all seinem Halbwissen eine ganze Nacht lang alleine verantwortlich für alle Notfälle und akuten Fragen. Die Oberärztin oder den Oberarzt ruft man ungern, weil man sie bzw. ihn nicht belasten will. Wer selbständig und alleine richtig entscheidet, wird geschätzt und vielleicht gelobt. Also muss man oft einsam die Verantwortung übernehmen und handeln. Das heisst, in der ersten, sehr prägenden Phase der beruflichen Weiterbildung wird man darauf trainiert, selbständig und ohne ein Team zu entscheiden. Und dieses Training hinterlässt sicher auch seine Spuren bei uns Ärztinnen und Ärzten. Als Kontrast dazu lernen wir tagsüber gezieltes, interprofessionelles Handeln mit verschiedenen Berufsgruppen. Und das hierarchische Weltbild in der Medizin? Die heutige, junge Generation hat gelernt, Informationen zum Beispiel mittels YouTube-Videos zu beschaffen und weniger die Vorgesetzten zu fragen. Aber nicht alles, was ein
Mensch durch langjährige Erfahrung lernt, kann im Netz gefunden werden. Hier setzt das neue Projekt Coach my Career an, welches in dieser SÄZ-Ausgabe auf Seite 334 beschrieben wird.

Wenn Kaderärztinnen und Kaderärzte zurücktreten, behandeln sie oft noch weiter Patienten. Aber all ihre Erfahrungen und über viele Jahre gesammeltes Wissen gehen der medizinischen Gemeinschaft verloren. In der Industrie wurde dies längst erkannt, und man hält die «Silberrücken» mit geeigneten Aufgaben im System. Die erfahrenen Kräfte werden zum Beispiel für Coaching-Aufgaben des jungen Kaders eingesetzt. So können Letztere in gemeinsamen Projekten von der erworbenen Erfahrung der Älteren profitieren. Das gleiche Grundprinzip verfolgt das Projekt Coach my Career. Erfahrenes, zurückgetretenes Ärzte-Kader hilft den jungen Ärztinnen und Ärzten bei deren Karriereentscheiden, indem sie ihnen Wege, Möglichkeiten und deren Auswirkungen aufzeigen. Das heisst, statt dass die Jungen alleine entscheiden müssen, bekommen sie Sparringpartner für diesen zentralen Planungsprozess. Am Projekt Coach my Career beteiligen

\section{Die Industrie hat «Silberrücken» längst ent-} deckt und nutzt ihre langjährige Berufserfahrung - Coach my Career tut dies in der Medizin.

sich sowohl die Spitalärzteschaft als auch Haus- und Kinderärzte. So können die jungen Kolleginnen und Kollegen durch diese gelebte Solidarität über die ärztlichen Generationen hinweg auf einer breiteren Basis ihre Zukunft besser planen.

Auch innerhalb der FMH steht das Projekt als wichtiges Zeichen für Zusammenarbeit und Solidarität über Generationen hinweg: Die SWIMSA, der VSAO, der VLSS, die mfe, das SIWF und das Departement stationäre Versorgung und Tarife haben das Projekt gemeinsam geplant und führen es zusammen durch - im Sinne einer geeinten Ärzteschaft. 